\title{
RESPONSIBLE LENDING - RESULTS AND PROSPECTS
}

\author{
RAMIN TSINARIDZE \\ Doctor of Business management, Assistant Professor \\ Batumi Shota Rustaveli State University, Georgia \\ ramin.tsinaridze@bsu.edu.ge
}

\section{LASHA BERIDZE}

Doctor of Business Administration, Associate Professor

St. Tbel Abuserisdze Teaching University, Georgia

I_beridze@mail.ru

Abstract. Research on financial sector activity is especially important for developing countries, including Georgia, as this sector is the main driving force of the countrys economy. Any economic reforms undertaken in the country have a significant impact on the general economic policy and economic growth.

Under the new regulation from January 1, 2019 the lending procedure from the financial sector has been tightened. At present time, they operate by the principle of responsible lending, which means, that a potential customer of a bank product should not be subject to a financial obligation that he or she will not be able to pay without financial difficulties.

The purpose of this study is to analyze the positive and negative effects of already existing responsible lending regulations, which is directing against indebtedness and to predict the expected future results. At the same time, the task of the research is to identify challenges, risks, results that accompany the reform based on the practices of developed and developing countries.

The study uses a combination of quantitative and qualitative methods. Also, in the research are used methods of system analysis, analogy, forecasting, synthesis, processing of statistical data and other methods. The methodological of the research is the fundamental articles of economic science. The paper widely used scientific and popular works by local and foreign authors, scientific studies, methodological publications and articles.

In the modern world any banking reform in developing and developed countries is linked to economic and political (often painful) changes, but this reform implementation is inevitable in all states, regardless of their level of development. The main reason for this is the reduction of indebtedness in the public and economic sectors, but this requires the introduction of a proper lending system that should not limit the development of the financial sector and, on the other hand, be an optimal source of replenishment of financial deficits.

Interest rate cuts are likely to have a short-term effect, which will not only help tighten lending policy by itself, and will require other macro-prudential decisions, especially when the NBG has raised its refinancing rate from $6.5 \%$ to $7 \%$, due to inflation targeting. It implements the monetary policy transmission mechanism and negatively impacts on the interest rate on loans issued by the banking sector and on aggregate demand itself, so economic growth is likely to be adjusted again.

KEYWORDS: RESPONSIBLE LENDING, INDEBTEDNESS, CREDITWORTHINESS, HEDGE BORROWER.

For citation: Tsinaridze, R., Beridze, L. (2020). Responsible Lending - Results and Prospects. Globalization and Business, №9, pp. 96-103 (In Georgian). https://doi.org/10.35945/gb.2020.09.012 


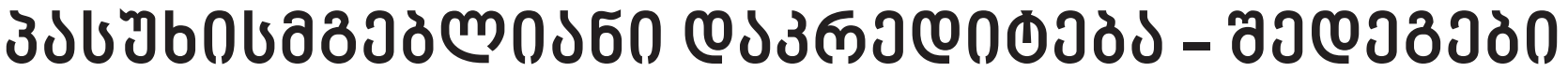

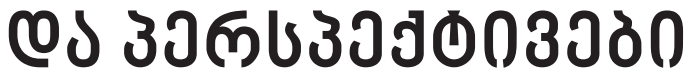

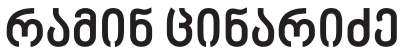

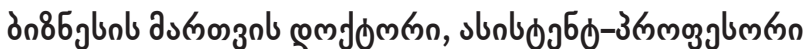

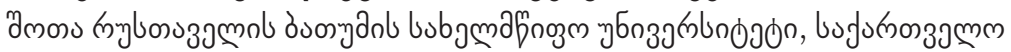

ramin.tsinaridze@bsu.edu.ge

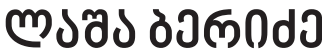

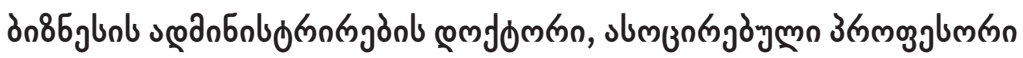

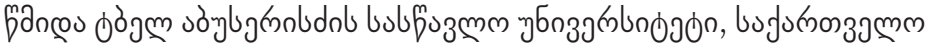

I_beridze@mail.ru

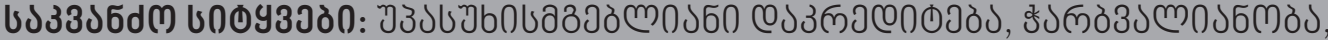

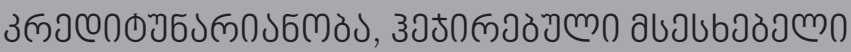

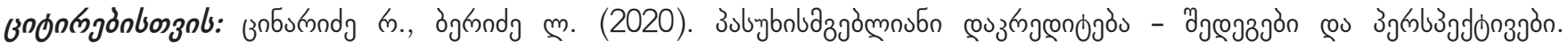

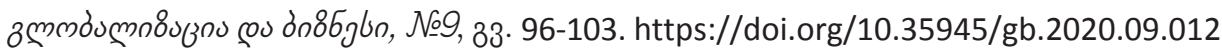

\section{ฆวเ১з১ल0}

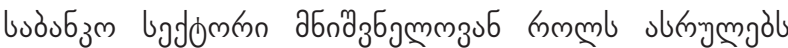

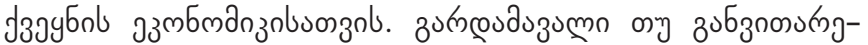

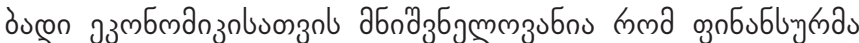

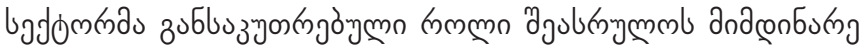

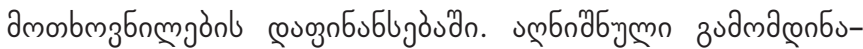

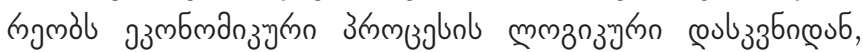

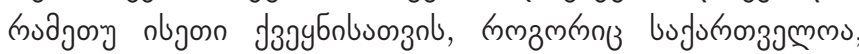

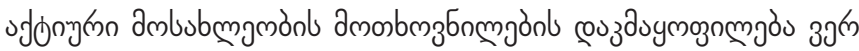

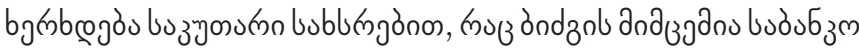

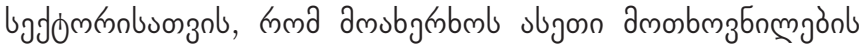

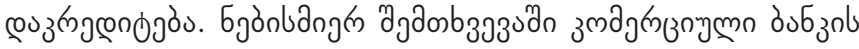

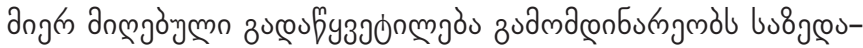

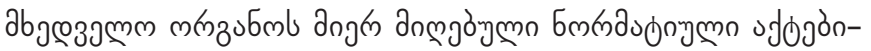

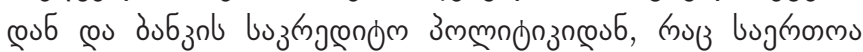

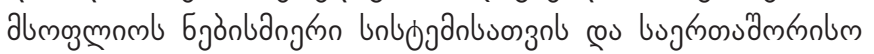

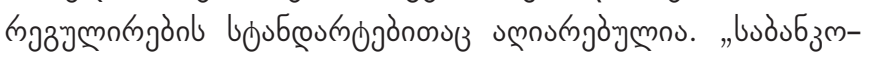

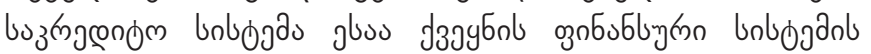

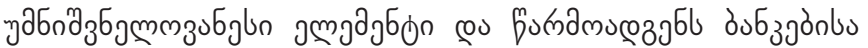

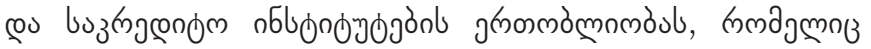

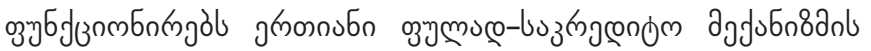

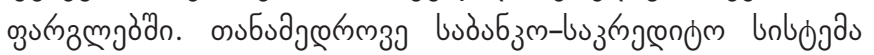

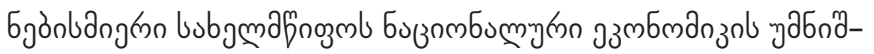

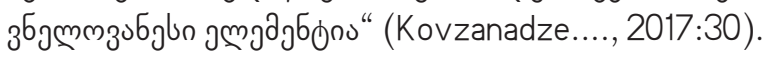

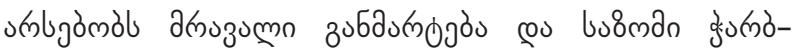

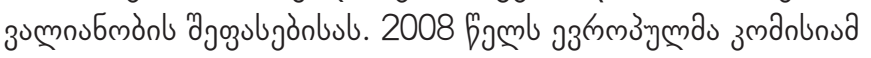

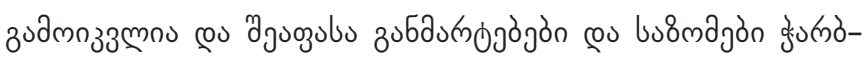

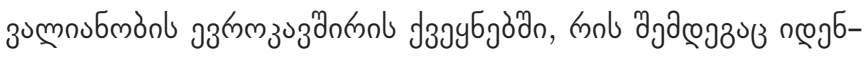

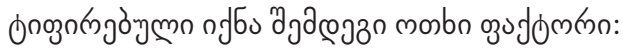

- эзмбмапзулю;

- commjònono;

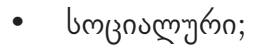

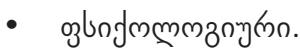

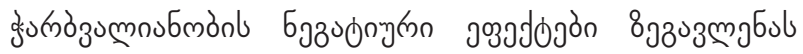

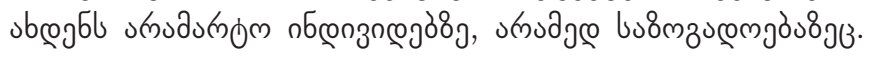

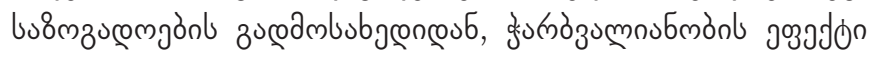

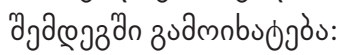

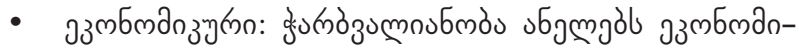

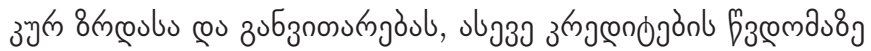

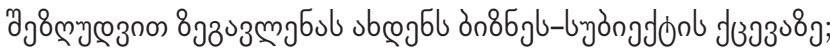

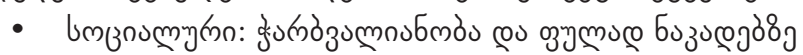

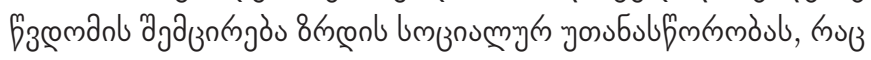

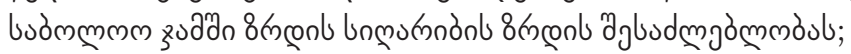

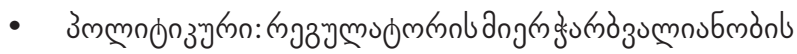

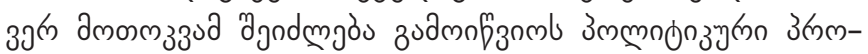

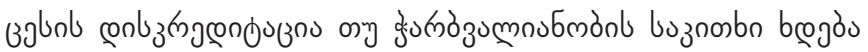

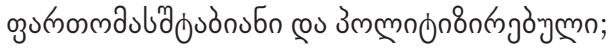

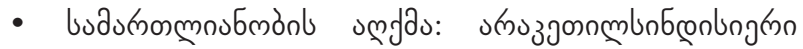
उ

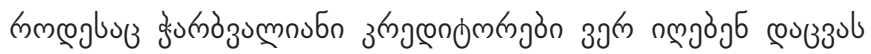
bubuas nonmmbuzub.

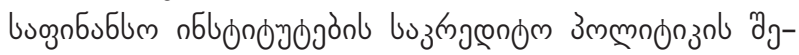

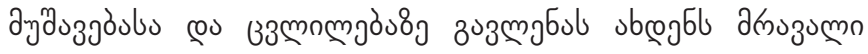




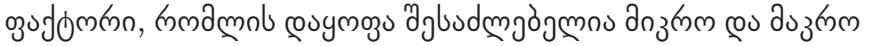

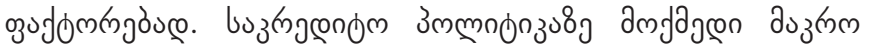

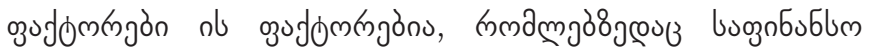

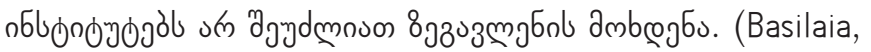
2018: 206)

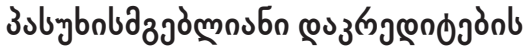

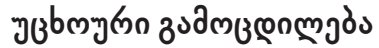

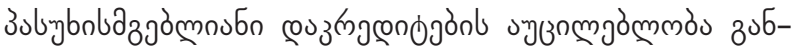

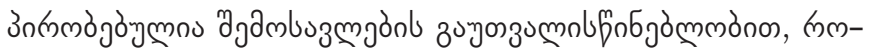

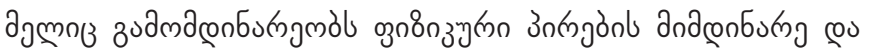

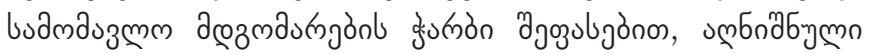

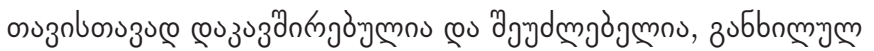

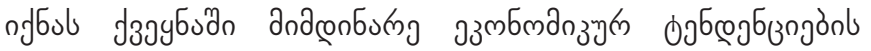

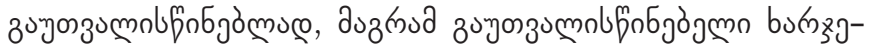

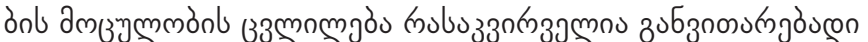

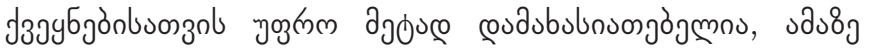

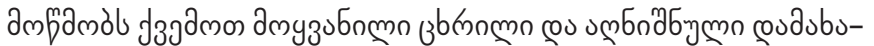

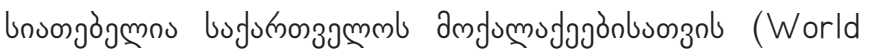
Bank..., 2013:11)

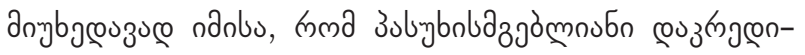

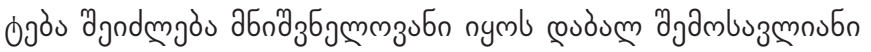

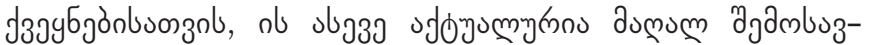

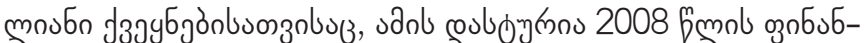

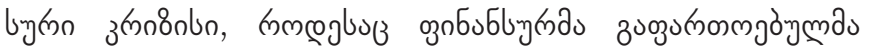

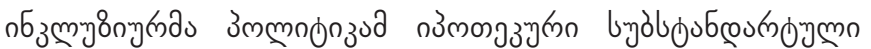

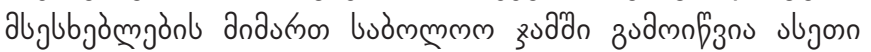

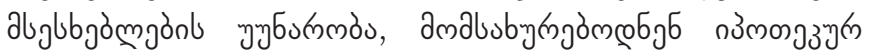

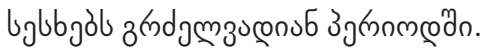

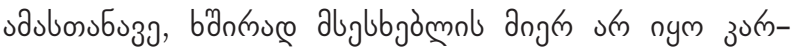

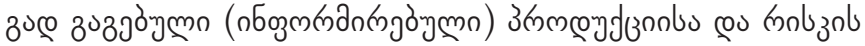

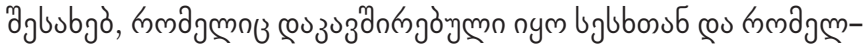

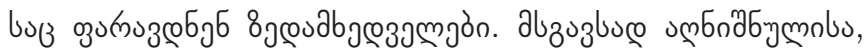

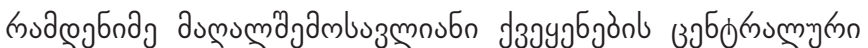

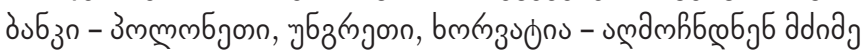

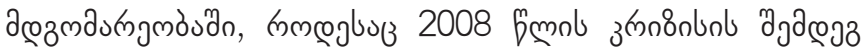

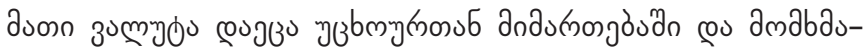

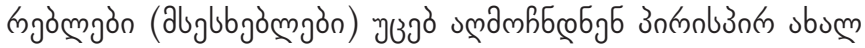

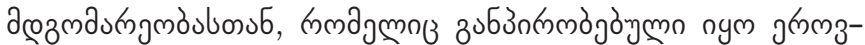

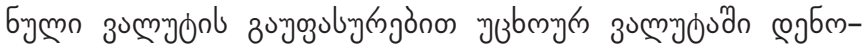

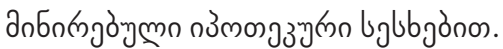

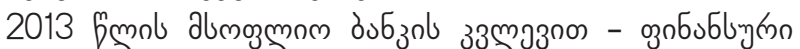

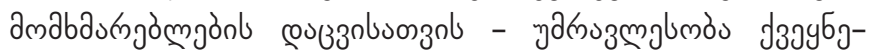

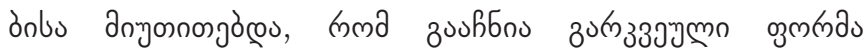

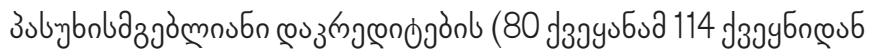

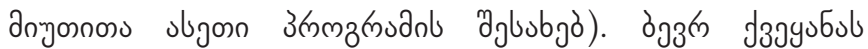

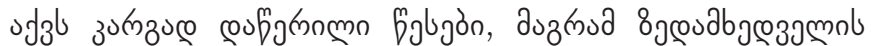

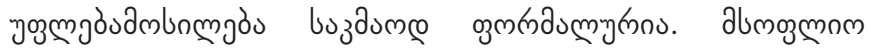
aubzol zзm

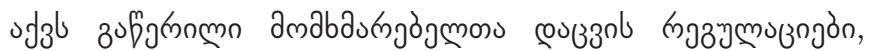

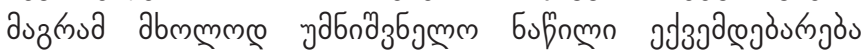

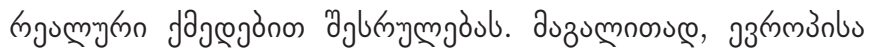

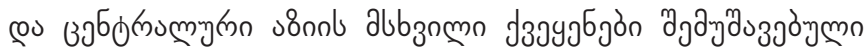

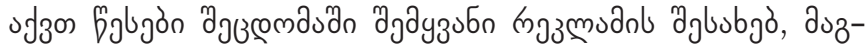

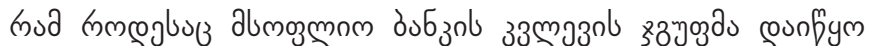

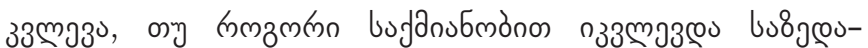

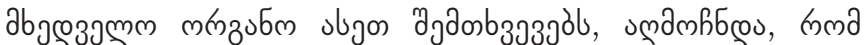

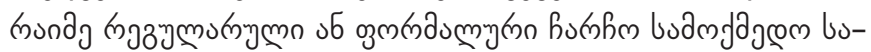

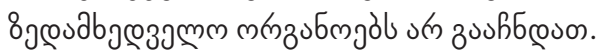

चб

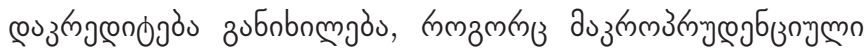

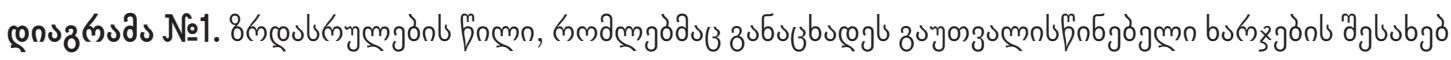

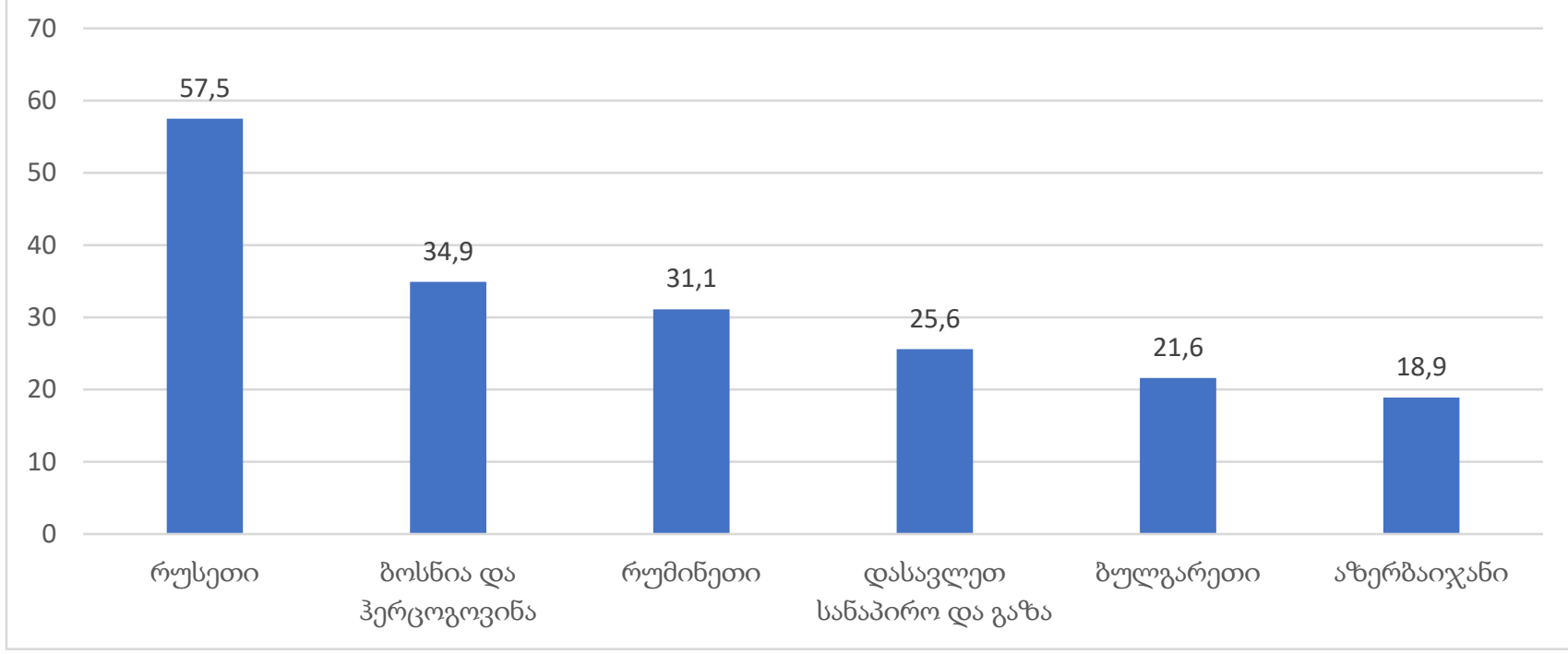

fyornm: World Bank 


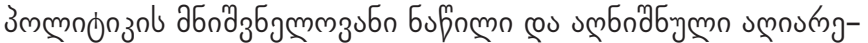

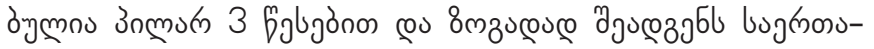

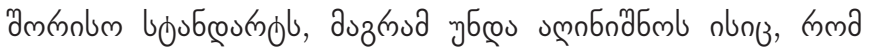

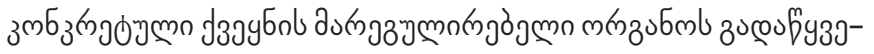

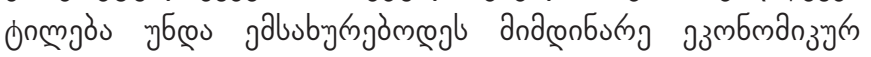

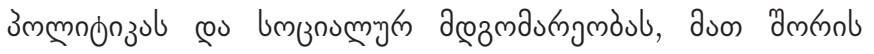

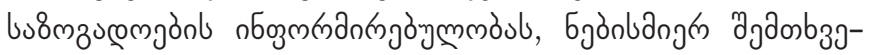

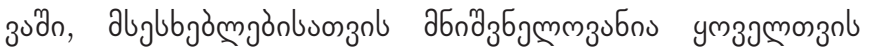

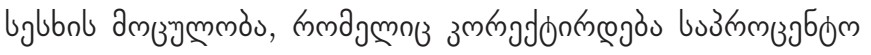

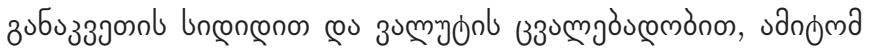

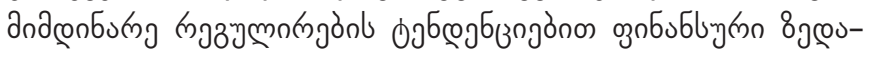

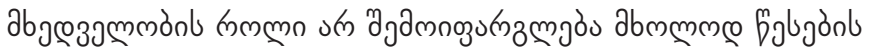

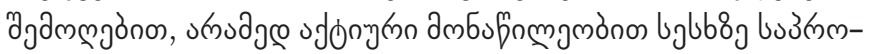

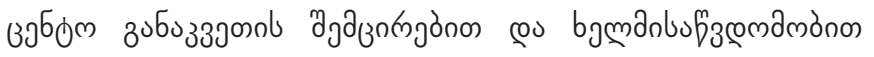
(World Bank.... 2013:26).

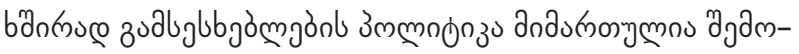

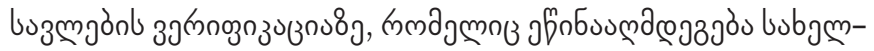

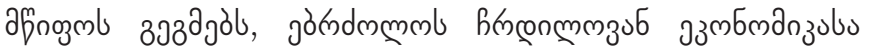

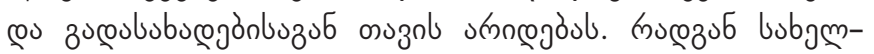

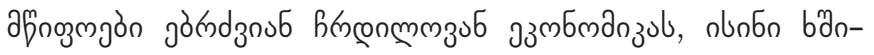

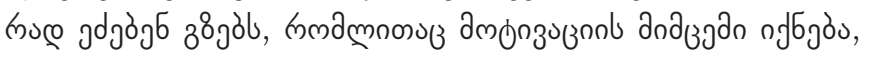

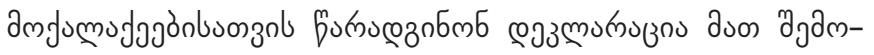

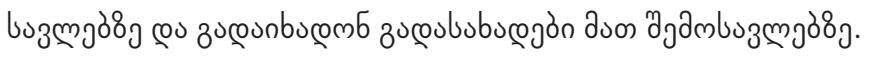

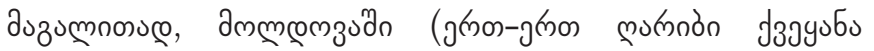

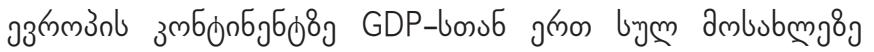

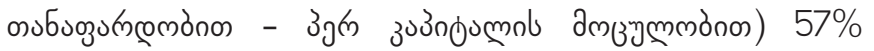

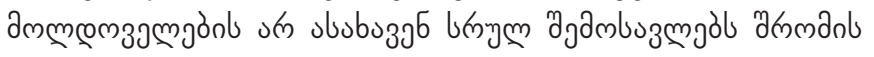

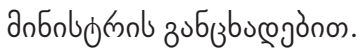

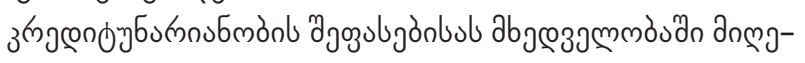

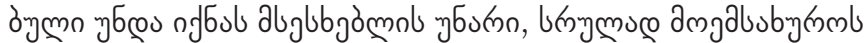

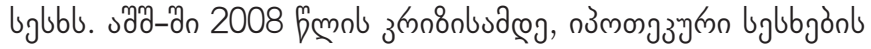

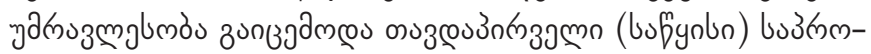

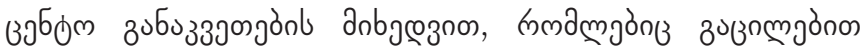

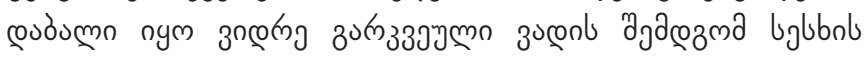

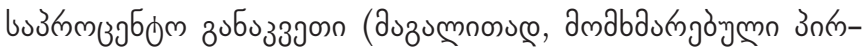

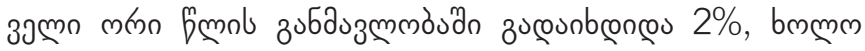

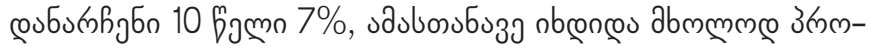
उ उदб J3aybnl (n

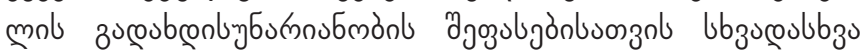

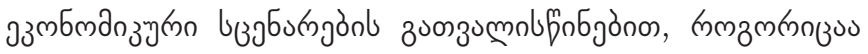

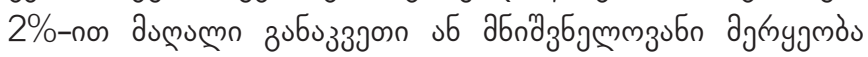

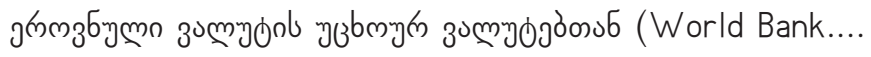
2013:25-38).

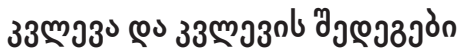

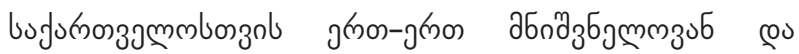

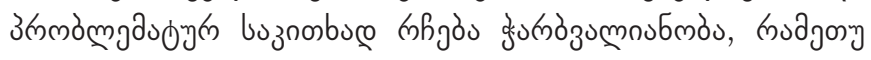

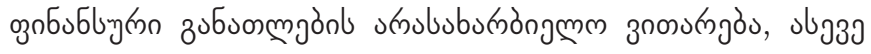

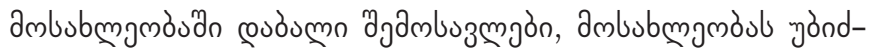

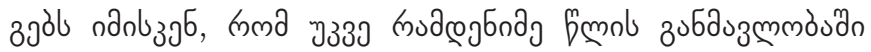

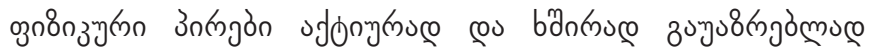

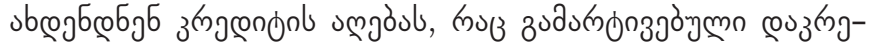

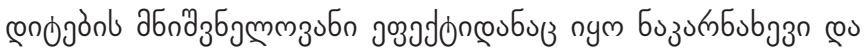

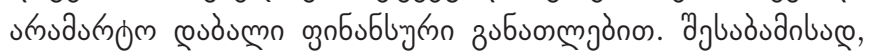

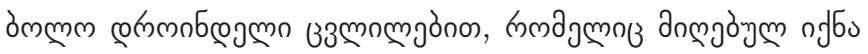

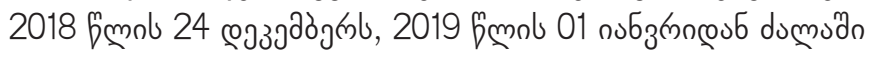

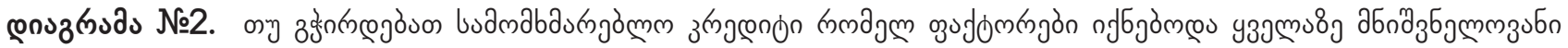

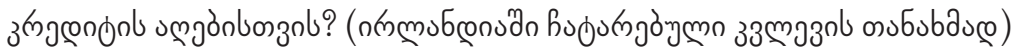

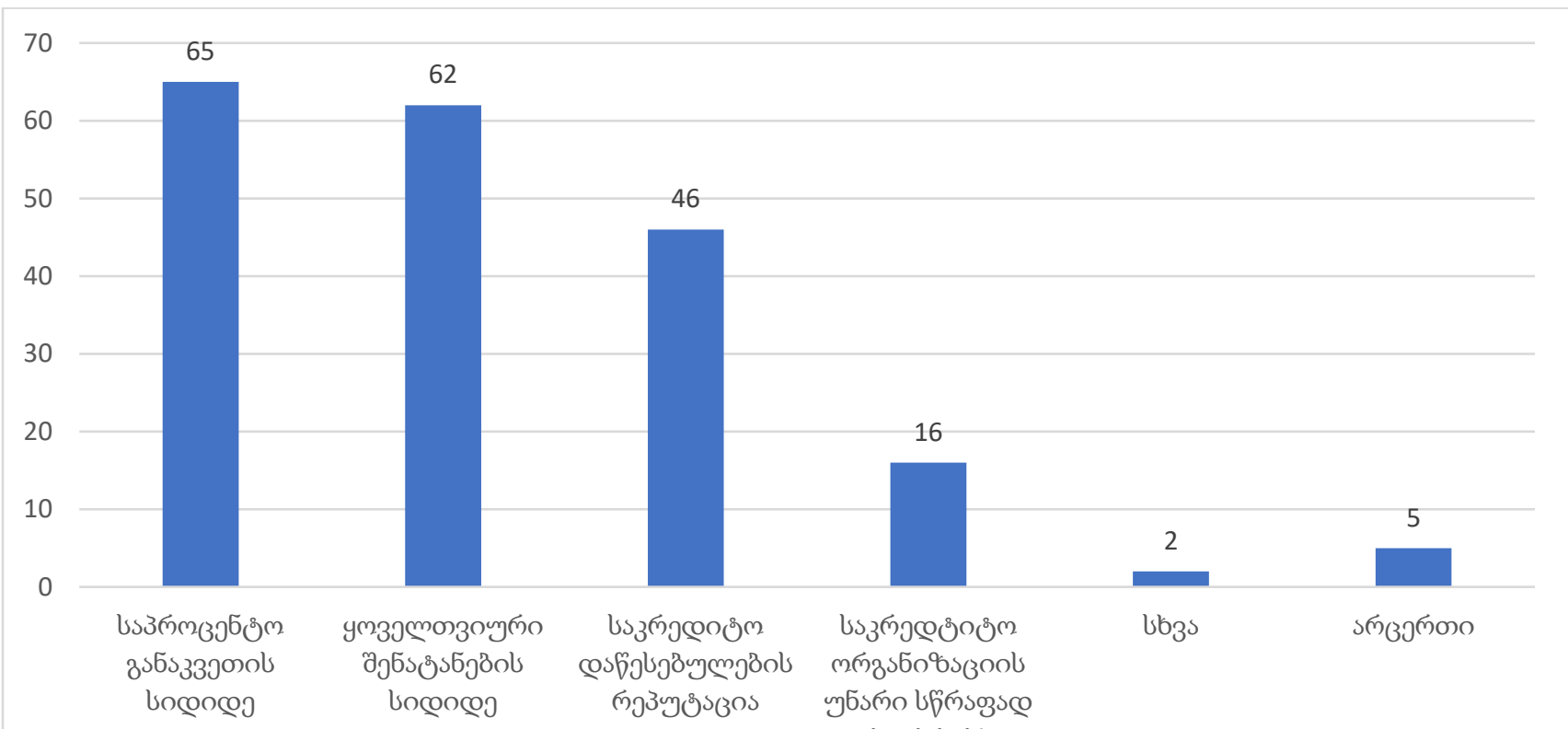

fyunn: World Bank 


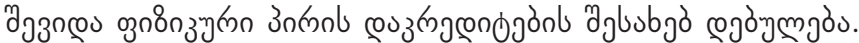

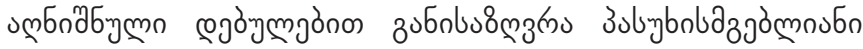

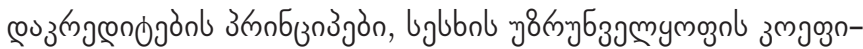

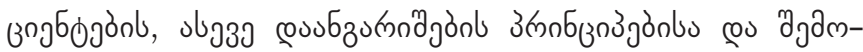

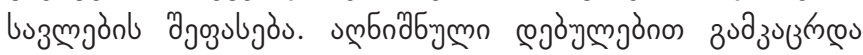

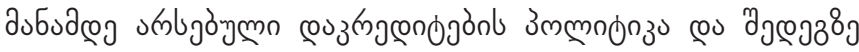

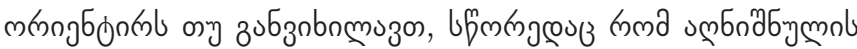

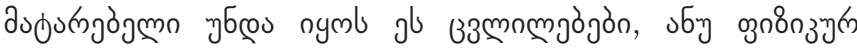
3n

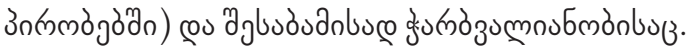

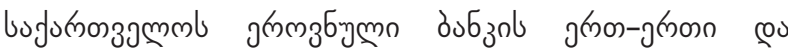

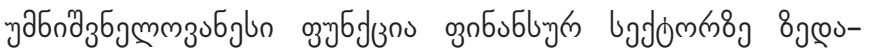

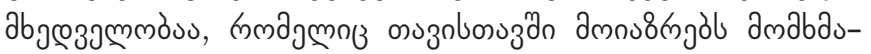

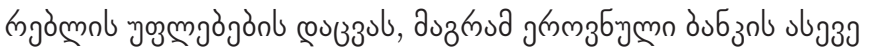

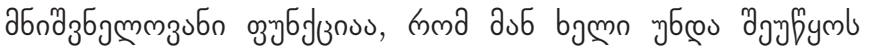

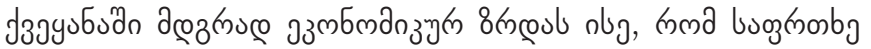

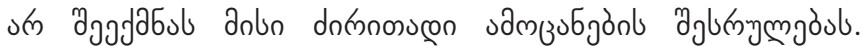

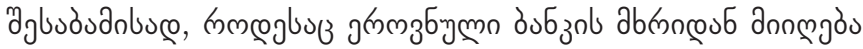

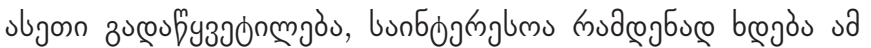

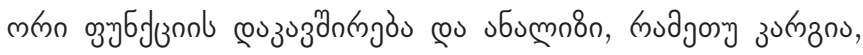

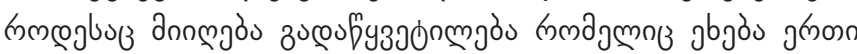

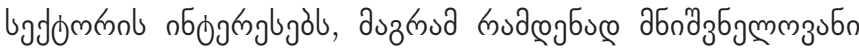

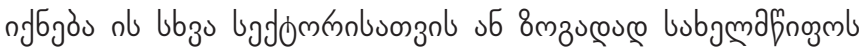
उзмбmanzolsonzol.

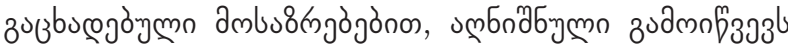

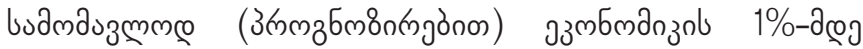

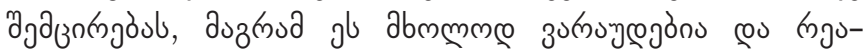

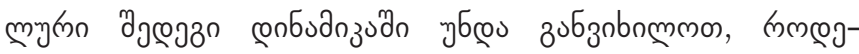

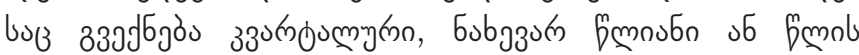

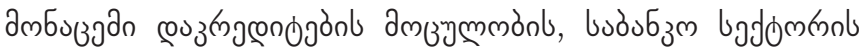

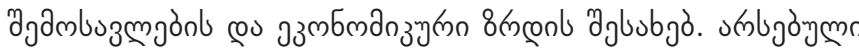

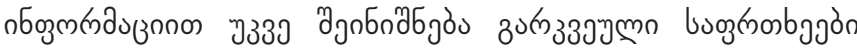

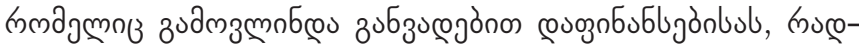

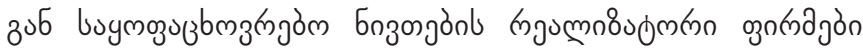

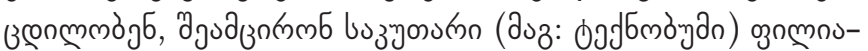

mgòn k num

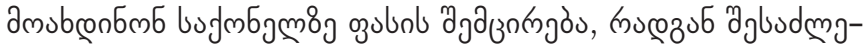

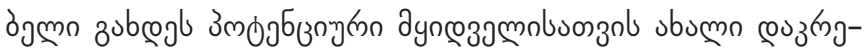

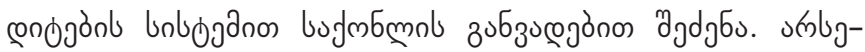

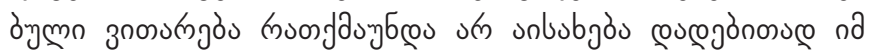

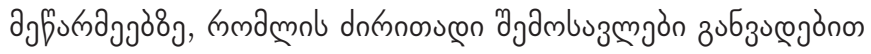
заупœз

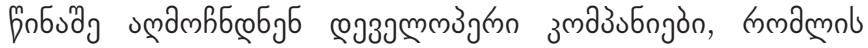

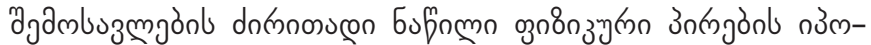

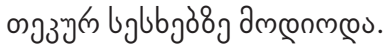

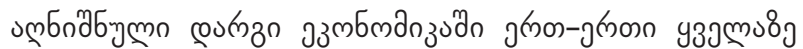

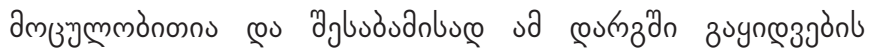

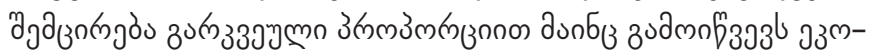

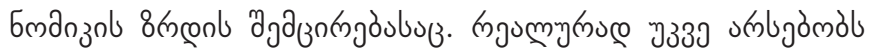

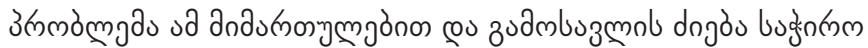

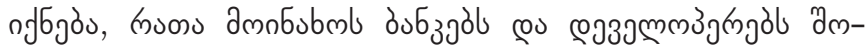

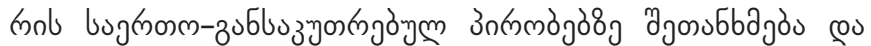

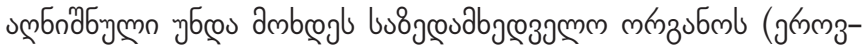

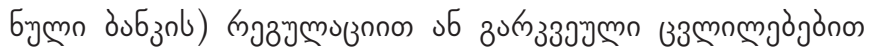
उ०Бмбам

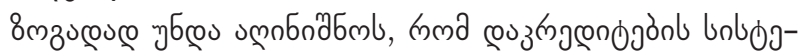

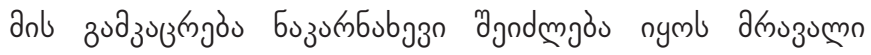

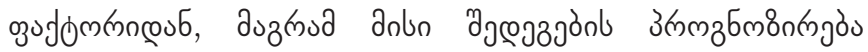

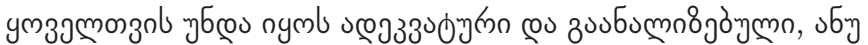

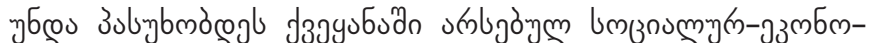

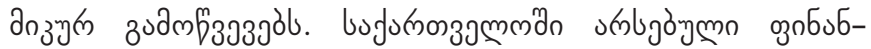

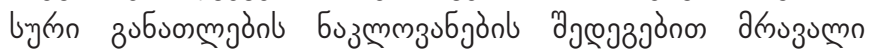

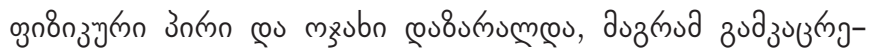

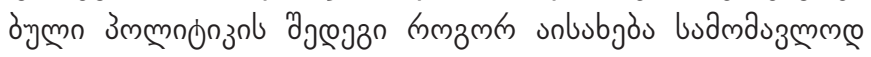

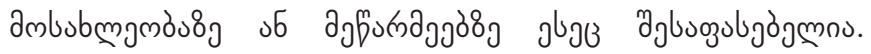

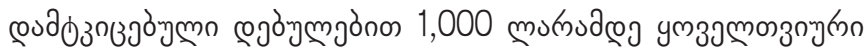

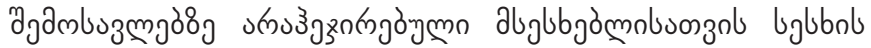

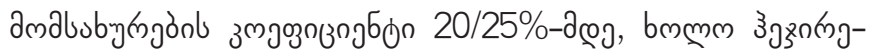

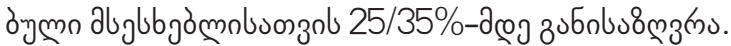

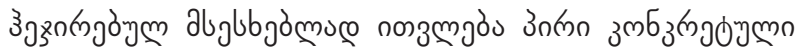

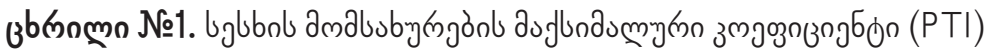

\begin{tabular}{|c|c|c|}
\hline 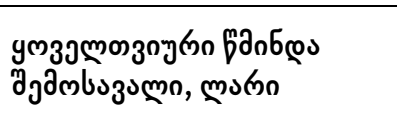 & 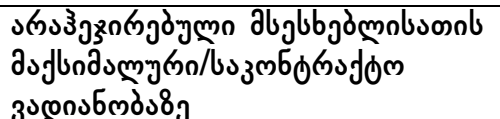 & 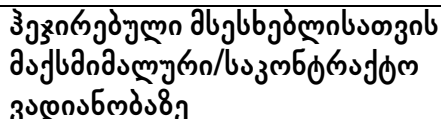 \\
\hline$<1,000$ & \multirow{2}{*}{$20 \% / 25 \%$} & $25 \% / 35 \%$ \\
\hline$<=1,000-2000<$ & & $35 \% / 45 \%$ \\
\hline$>=2,000-4,000<$ & $25 \% / 30 \%$ & $45 \% / 55 \%$ \\
\hline$>=4,000$ & $30 \% / 35 \%$ & $50 \% / 60 \%$ \\
\hline
\end{tabular}

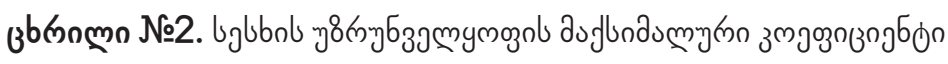

\begin{tabular}{|c|c|}
\hline 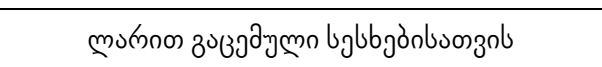 & $85 \%$ \\
\hline 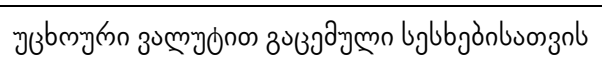 & $70 \%$ \\
\hline
\end{tabular}


3umyonl

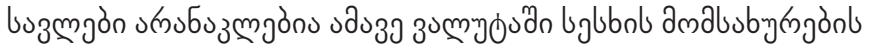

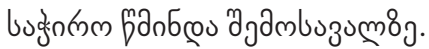

ug

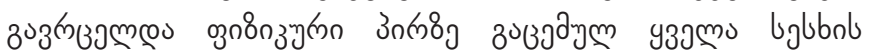

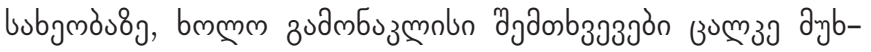

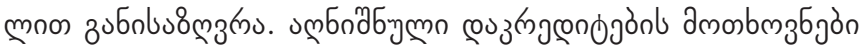

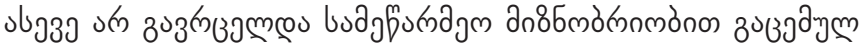
bglbgabg.

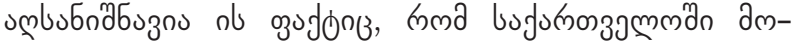

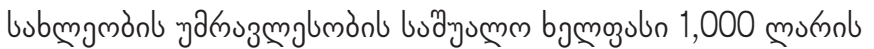

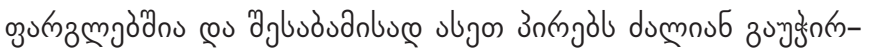

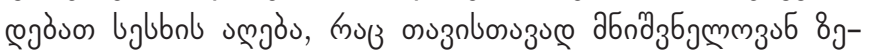

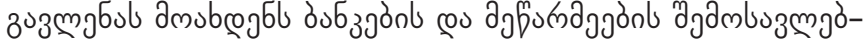

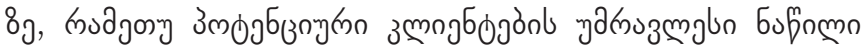

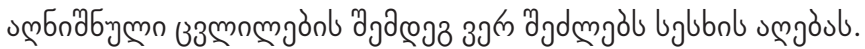

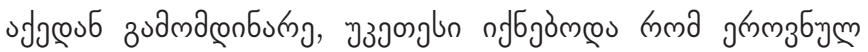

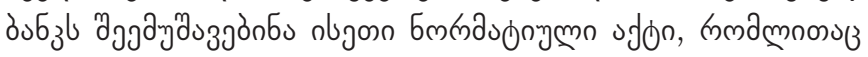

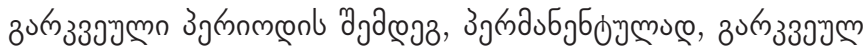

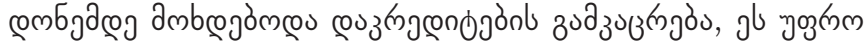

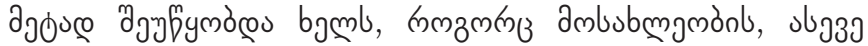

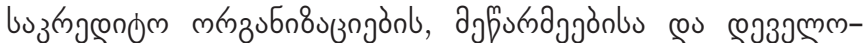

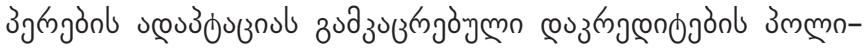
onз

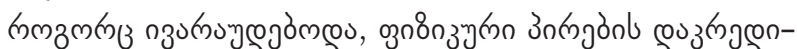

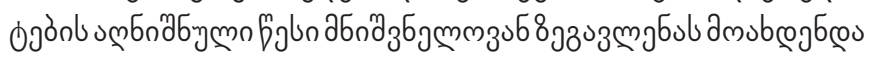

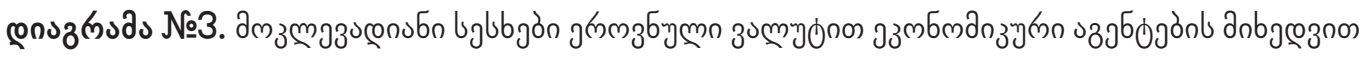

anmombn mukno

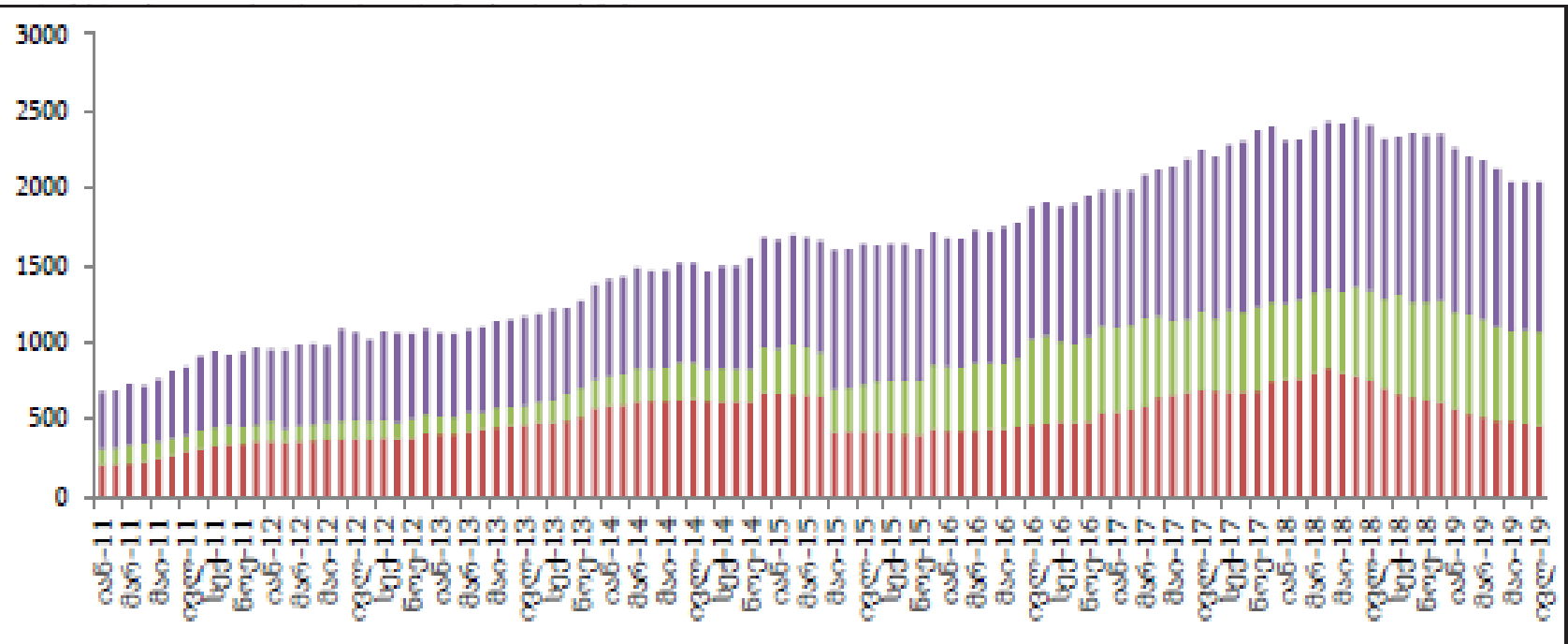

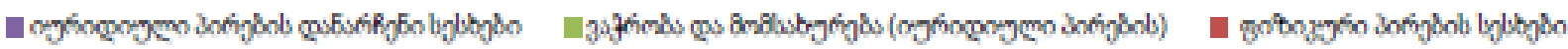

fyutrm: National Bank of Georgia

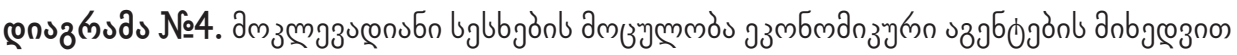

domambo mukno

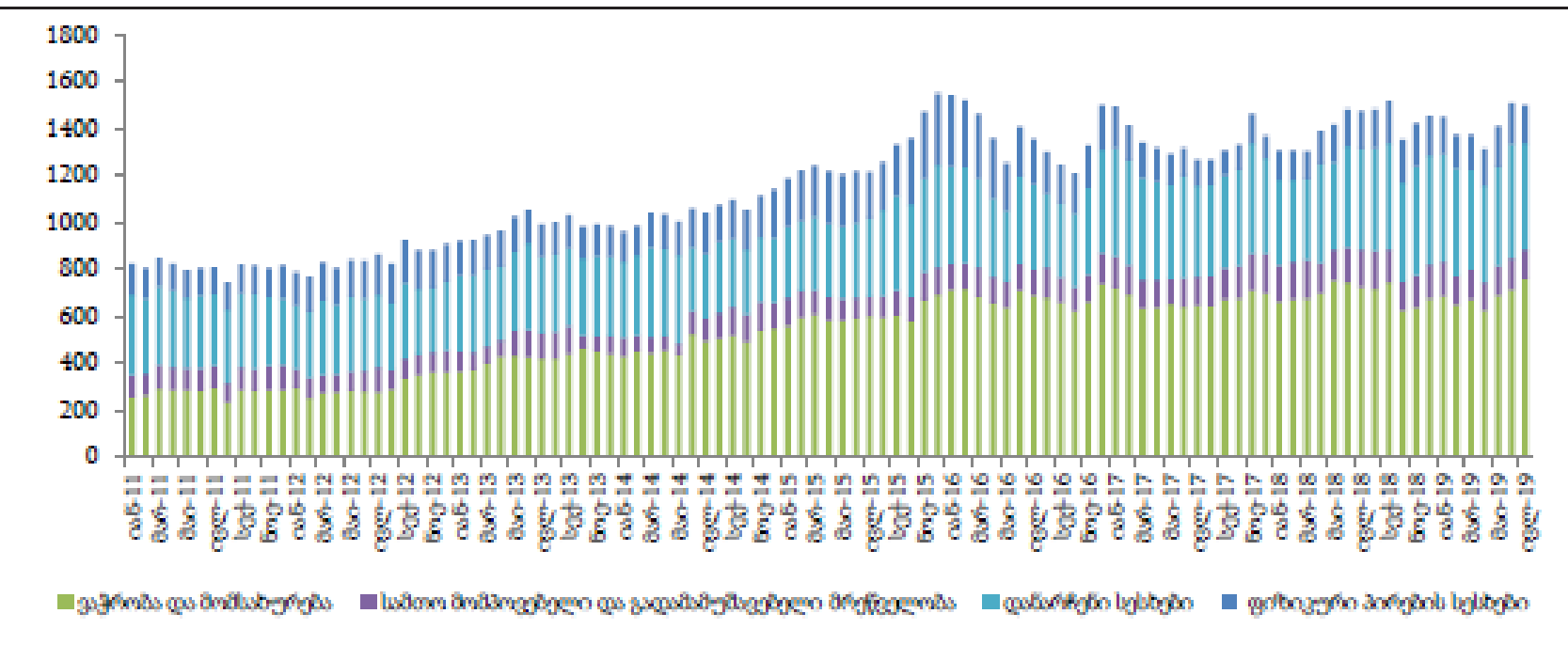

fyutrom: National Bank of Georgia 


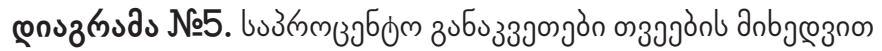

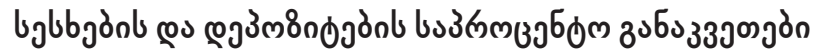

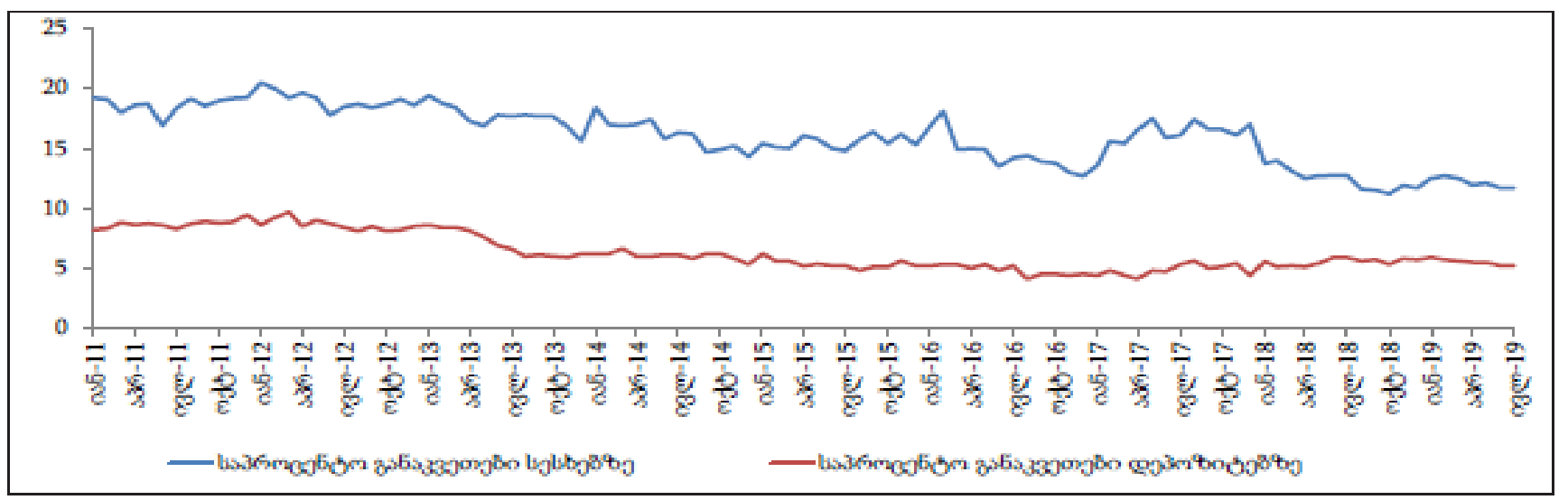

fyumm: National Bank of Georgia

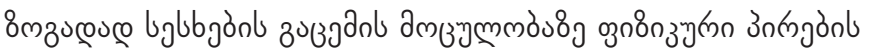

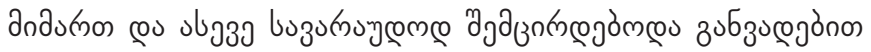

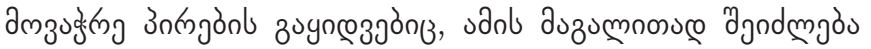

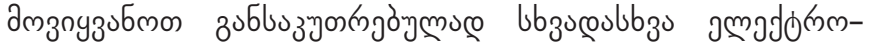

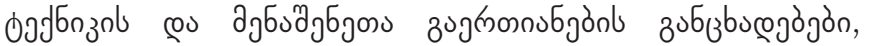

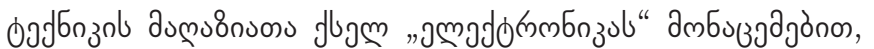

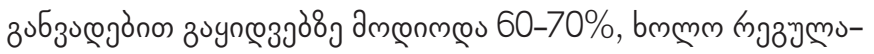

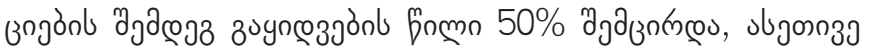

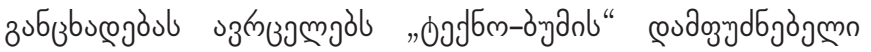

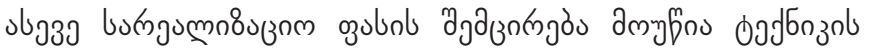

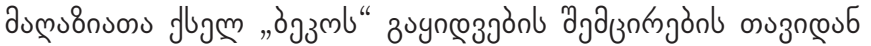

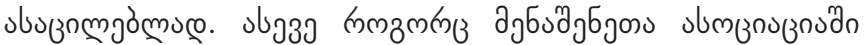
u u

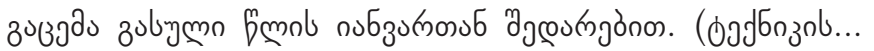
:2019)

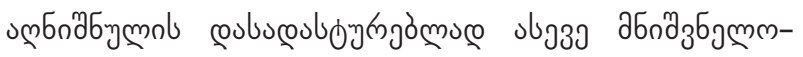

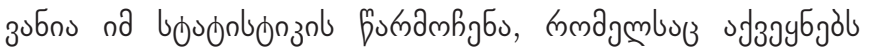

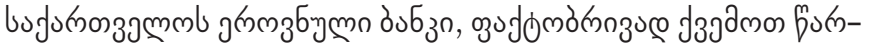

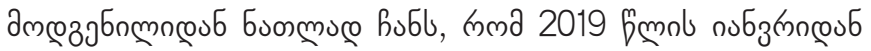

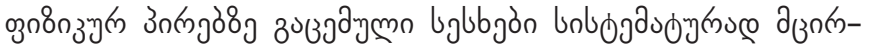

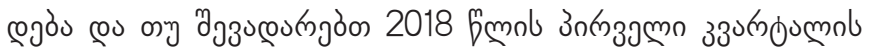

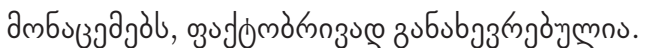

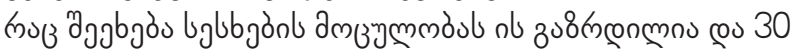

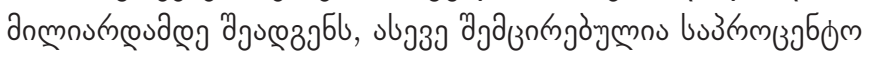

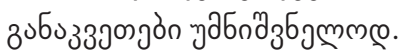

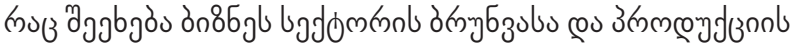

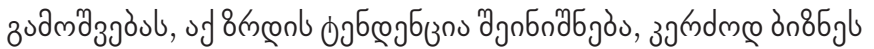

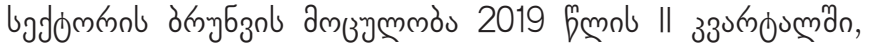

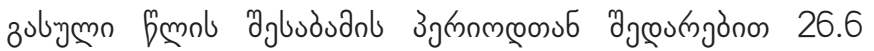

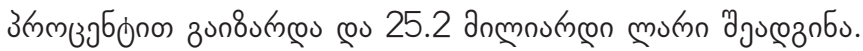

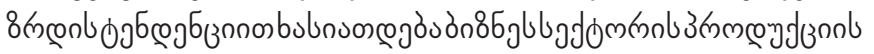

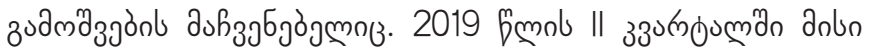

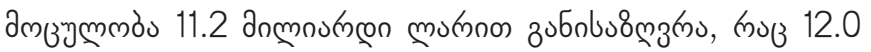

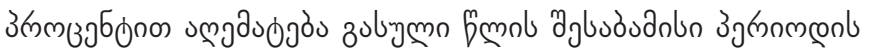
дı

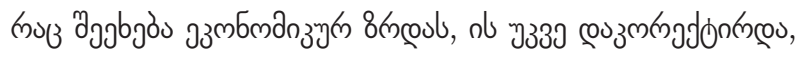

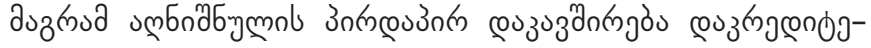

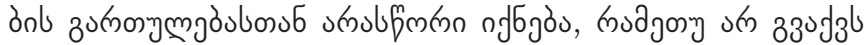

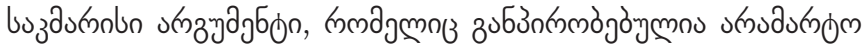

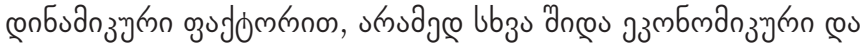

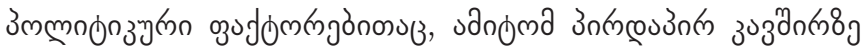

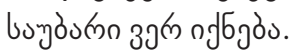

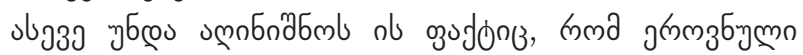

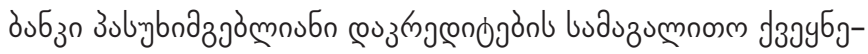

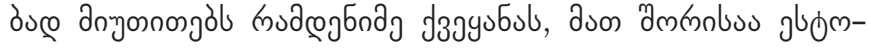

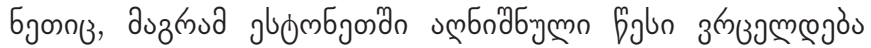

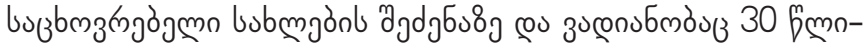

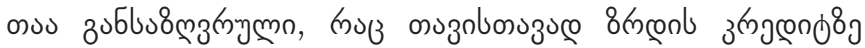

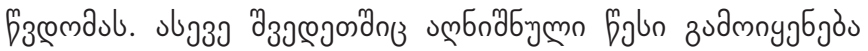

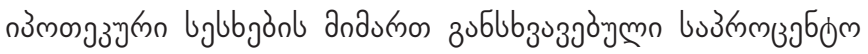

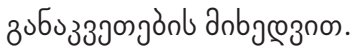

\section{๒১৬з3ธ000 бง60ल0}

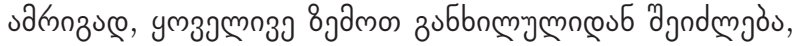

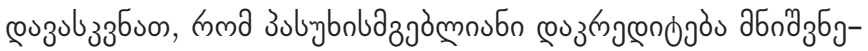

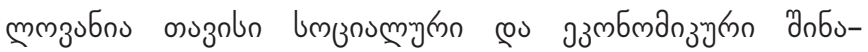

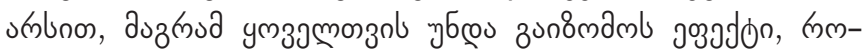

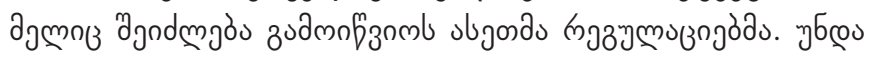
u мnб

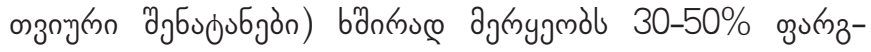

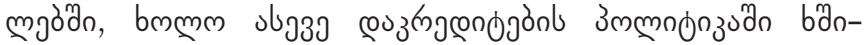

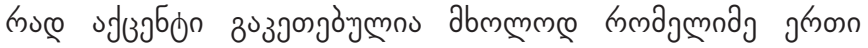

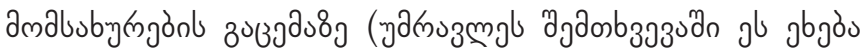

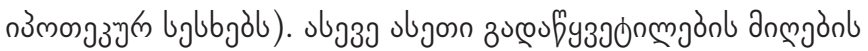

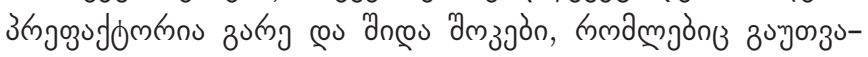

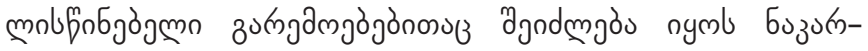

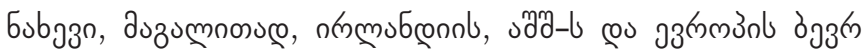

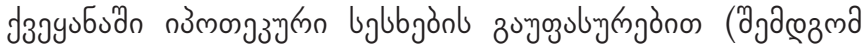

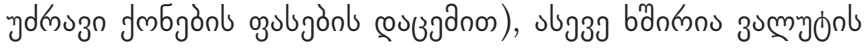




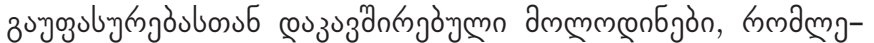

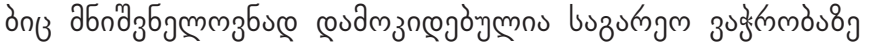

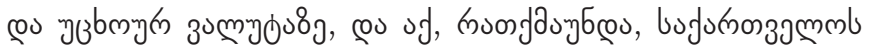

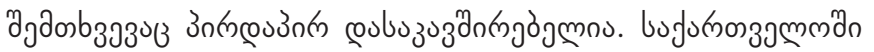

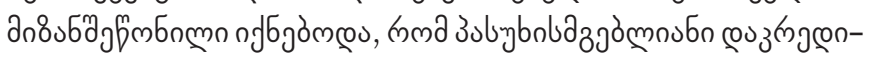

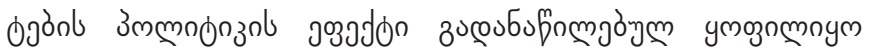

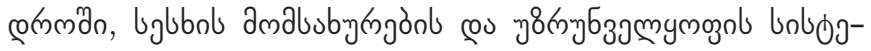

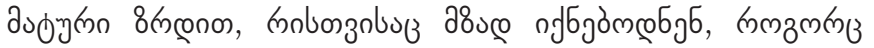

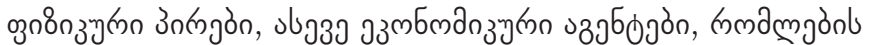

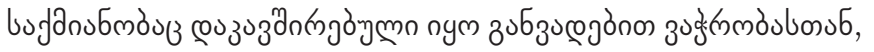

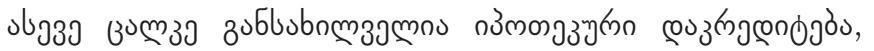

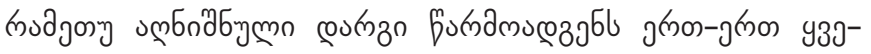

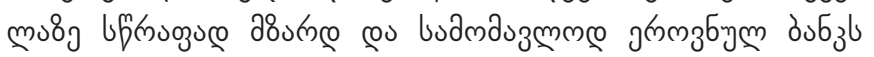

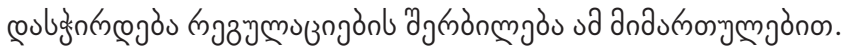

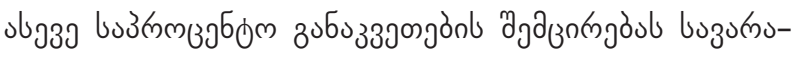

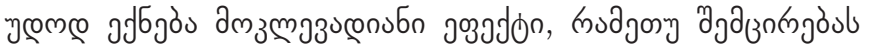

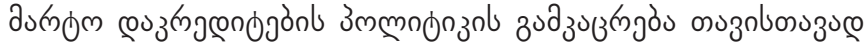

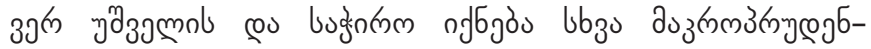

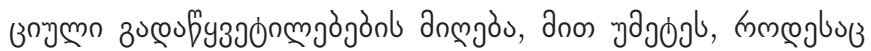

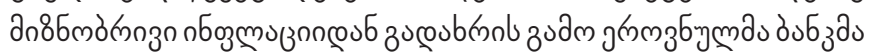

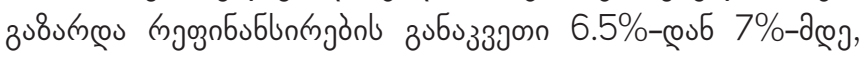

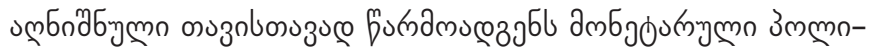

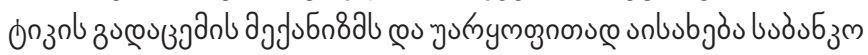

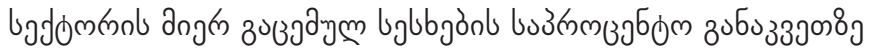

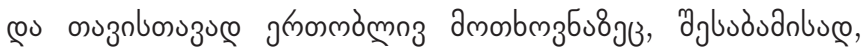

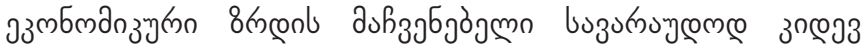

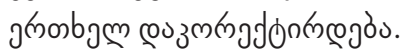

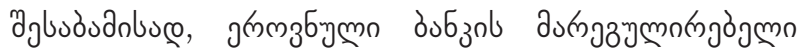

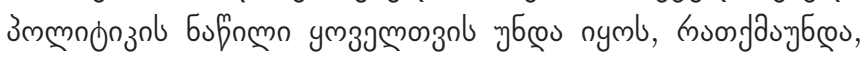

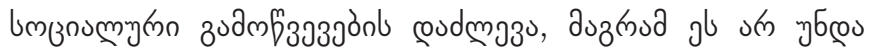

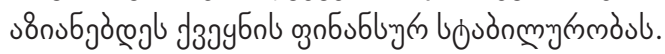

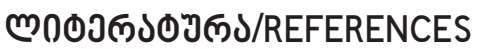

Kovzanadze I., kontridze G. (2017). Modern Banking: Theory and Practice. Tbilisi (in Georgian).

Basilaia K. (2018). Banking. Tbilisi (n Georgian).

Kokiauri L (2012). Basics of Banking. Tbilisi (In Georgian).

Tsaava G., Khantadze G. (2015). Banking Management (Theory, Methods and Practice). Manual. Tbilisi (In Georgian).

Gelashvili M. (2018) Risk Management in Commercial Banks. Journal of Innovative Economics and Management, №2 (In Georgian).

Order of the President of the National Bank of Georgia №281/04. (2018) December 24. (Available: 09.09.2019) (In Georgian). National Bank of Georgia. Review for July. (2019). (Available: 09.09.2019) (In Georgian).

National Statistics Office of Georgia, 2nd Quarter of 2019. (2019) Review. (Available: 09.09.2019) (In Georgian).

World Bank, Overview of Supervisory Regulations, October 2013. (2013) (available: 09.09.2019).

House technic stores are with microfinance organizations on a new installment scheme. (2019). (Available: 09.09.2019) (In Georgian). 